\title{
The TNT equivalence of an optimum propane-oxygen mixture
}

\author{
J M Dewey \\ Department of Physics and Astronomy, University of Victoria, Victoria, BC, \\ V8N 2A4, Canada \\ E-mail: jdewey@uvic.ca
}

Received 10 May 2005, in final form 6 September 2005

Published 18 November 2005

Online at stacks.iop.org/JPhysD/38/4245

\begin{abstract}
Measurements of the times of arrival of the primary shock produced by the explosion of a nominal 20 tn propane-oxygen mixture have been analysed to provide the variation of the peak hydrostatic overpressure as a function of distance. The results have been scaled to those for a charge of unit mass at normal temperature and pressure, based on the masses of the propane and oxygen and of the propane alone. The scaled results are compared with those produced by the explosion of a hemispherical unit mass of TNT to provide the TNT equivalence factor as a function of overpressure and distance. For overpressures greater than $1 \mathrm{~atm}$ there is a strong dependence on the distance from the centre of the explosion, but at lower overpressures the equivalence factors have almost constant values of 0.55 for the propane-oxygen mixture and 1.95 for the propane alone. The significance of these findings, in relationship to vapour cloud explosions and boiling liquid expanding vapour explosions, is discussed.
\end{abstract}

\section{Introduction}

The blast waves produced by the detonation of different types of explosives are usually compared with the blast wave produced by an equivalent mass of TNT. There are a number of reasons for doing this. The main one is that more information is available about the physical properties of the blast waves produced by TNT, as a free air, surface-burst or height-ofburst explosion, than for any other explosive. Knowing the energy yield of an explosive is not by itself sufficient to determine the properties of the resulting blast wave because not all the energy may be released in the compression wave. For example, in the case of a nuclear explosion approximately half of the available energy is released in the blast wave and the remainder as nuclear and electro-magnetic radiations. At the other extreme, a bursting balloon will release most of its energy into a compression wave.

The rate at which the energy is released also has a significant effect on the properties of the resulting blast wave. Two explosives releasing the same amount of compressional energy, but at different rates, will produce blast waves with different properties. A rapid detonation will generate an initially intense blast wave with a strong primary shock, across which there will be large changes of entropy. As a result, less energy is available as the blast expands to lower overpressures. A less intense detonation, or a deflagration, will produce a weaker initial shock with smaller changes of entropy so that more energy is available in the blast at greater distances.

Kleine et al (2003) showed that the TNT equivalence of an explosive may not be single valued, but a function of the distance from the centre of the explosion. For the explosion of gaseous mixtures, most sources quote a single value for the TNT equivalence. The single equivalence factor may have been derived by comparing the energy yield of an explosive with that for the same mass of TNT, as is explained, for example, by Baker et al (1983). Alternatively, the factor can be obtained by comparing the distances from the charges at which various blast effects or measurements are observed for the candidate explosive and for TNT. This method results in variations of typically more than $20 \%$ in the equivalence factor for an explosive (e.g. Cooper (1996)), probably because the measurements and observations were made at different scaled distances.

Determining the TNT equivalence factor for a BLEVE (boiling liquid expanding vapour explosion) is even more difficult. A typical scenario for a BLEVE is as follows. A rail or road tanker for the transportation of a fuel, such as propane, becomes involved in an accident that causes a leakage of the 
fuel. The leaked fuel may ignite and cause a fire that envelops the tanker. The fire heats the liquid within the tanker to boiling point and may further damage the tanker itself. These effects may be sufficient to explosively rupture the casing of the tanker. The initial explosion normally causes a minimal blast wave but may project fragments of the container to large distances and disburse the fuel as a droplet and vapour cloud. The fuel will mix with air and may reach a flammable or even detonable concentration, such that a second, and much more powerful, unconfined vapour-cloud explosion (VCE) occurs.

The efficiency of such an explosion, based only on the amount of fuel available, is difficult to determine. Baker et al (1983) suggest an efficiency of $10 \%$ for such an explosion. The difficulties associated with the determination of an appropriate TNT equivalence factor for BLEVEs and VCEs are described by Birk et al (1995), Birk (1996) and Martinsen and Melton (1999a, 1999b). Because of these difficulties, some agencies have developed alternative models to describe VCE that do not rely on a comparison with TNT, e.g. Van den Berg et al (1991). However, this may make it more difficult to assess the damage and injury potential of the blast waves, since these features are commonly linked to the properties of blast waves from TNT.

Some of the difficulties discussed above arise because the variations of the blast wave properties as functions of distance for a BLEVE or VCE are likely to be very different from those for a high explosive, such as TNT. A search of the literature revealed few results for BLEVEs and VCEs in which accurate measurements of blast wave properties had been made over a wide range of distances from close to the explosive source to the low-pressure region. An exception to this was a large hemispherical explosion of a stoichiometric mixture of propane and oxygen, carried out at Suffield, Canada, in 1966, and known in Canada as FE567/2a and in USA as Operation DISTANT PLAIN Event 2a. The blast measurements from this experiment were reported by Anderson (1970), and a later analysis was carried out by Dewey and McMillin (1981). Neither of these publications reports a determination of the TNT equivalence of the explosion as a function of distance from the source nor relates the properties directly to the masses of propane and oxygen that were used. The present study was carried out to rectify these deficiencies and to provide the TNT equivalence as a function of distance for an optimum propane/oxygen explosion.

\section{Explosive source}

In the late 1950 s and throughout the 1960s, an extensive series of experiments was undertaken at Suffield, Canada, to study the physical properties of blast waves produced by spherical and hemispherical charges of TNT, ranging in mass from $25 \mathrm{~kg}(60 \mathrm{lb})$ to 500 tonne. An alternative explosive was sought because of the relatively high cost of using TNT. One suggested alternative was to use gaseous explosions involving stoichiometric mixtures of propane or methane with oxygen. The resulting series of experiments was known in Canada as FE567 or as Operation DISTANT PLAIN in USA.

The first experiment of this series involved a $20 \mathrm{tn}$ (US short ton $=907.19 \mathrm{~kg}$ ) sphere of TNT, which was successfully detonated at the top of a $25 \mathrm{~m}$ tower. The second experiment was to have involved a spherical balloon, $33.5 \mathrm{~m}$ diameter, filled with a mixture of methane and oxygen and tethered with its centre $25 \mathrm{~m}$ above ground. While it was being filled there was a structural failure of the balloon and a large volume of oxygen was spilled resulting in a fire that seriously injured one person. Experiment 2a, the one analysed here, used a hemispherical balloon, $38 \mathrm{~m}$ diameter, filled with a stoichiometric mixture of propane and oxygen with a nominal total mass of $20 \mathrm{tn}$. The filling process was extremely difficult due to the wind conditions, and as a result the centre of the balloon was displaced approximately $3 \mathrm{~m}(10 \mathrm{ft})$ from its planned position. This charge was successfully detonated on 22 July 1966. As a result of the difficulties and hazards experienced in carrying out these experiments, no further large scale gaseous explosions were attempted and an alternative solid explosive, ammonium nitrate-fuel oil (ANFO), was used to replace TNT in large scale blast experiments.

No records could be found of the exact amounts of propane and oxygen that were used in the hemispherical gas-bag experiment. The dimensions and composition of the charge, given by Anderson (1970), are as follows. The diameter of the hemispherical bag was $125 \mathrm{ft}$. The gas mixture ratio was 3.5 moles of oxygen per mole of propane. The internal overpressure was $0.44 \mathrm{in}$. of water. The ambient atmospheric pressure was $13.7 \mathrm{psi}$. The external ground temperature was $118 \mathrm{~F}$ and the air temperature at a height of $6 \mathrm{ft}$ was $74 \mathrm{~F}$. This information was used to calculate the masses of propane and oxygen as follows.

The overpressure in the bag, $\Delta P$, was 0.44 in. of water. Therefore,

$$
\begin{aligned}
\Delta P= & 0.44 \text { in. } \times 2.54 \times 10^{-2} \mathrm{~m} \mathrm{in.}^{-1} \times 9.81 \mathrm{~m} \mathrm{~s}^{-2} \\
& \times 0.9979 \times 10^{3} \mathrm{~kg} \mathrm{~m}^{-3} \\
= & 109.4 \mathrm{~Pa},
\end{aligned}
$$

where the density of water is $997.9 \mathrm{~kg} \mathrm{~m}^{-3}$ and the gravitational acceleration is $9.81 \mathrm{~m} \mathrm{~s}^{-2}$. The atmospheric pressure, $P_{0}$, was $13.71 \mathrm{psi}=94527 \mathrm{~Pa}$. Therefore, the absolute pressure in the bag, $P=94636 \mathrm{~Pa}$. It was assumed that the temperature, $T$, of the gas in the bag was $74 \mathrm{~F}=296.48 \mathrm{~K}$. The diameter of the bag was $125 \mathrm{ft}=38.1 \mathrm{~m}$. Therefore, the volume, $V$, was $14479 \mathrm{~m}^{3}$. The number of moles of gas, $n$, is given by

$$
n=\frac{P V}{R T}=\frac{94636 \times 14479}{8.3145 \times 296.48}=555867 \text { mole }
$$

where $R$ is the universal gas constant. The molar ratio of oxygen to propane was 3.5 . Therefore,

$$
\frac{3.5}{4.5} \times 555867=432341 \text { mole of oxygen }
$$

and

$$
\frac{1}{4.5} \times 555867=123526 \text { mole of propane. }
$$

Using the molar mass of oxygen $=31.9988 \mathrm{~g}$, and of propane $=44.097 \mathrm{~g}$, gives the mass of oxygen $=13834 \mathrm{~kg}$ and propane $=5447 \mathrm{~kg}$. Therefore, the total mass of the charge was $19281 \mathrm{~kg}$. The nominal mass of the charge was $20 \mathrm{tn}=18144 \mathrm{~kg}$. Figure 1 shows the charge before detonation. 


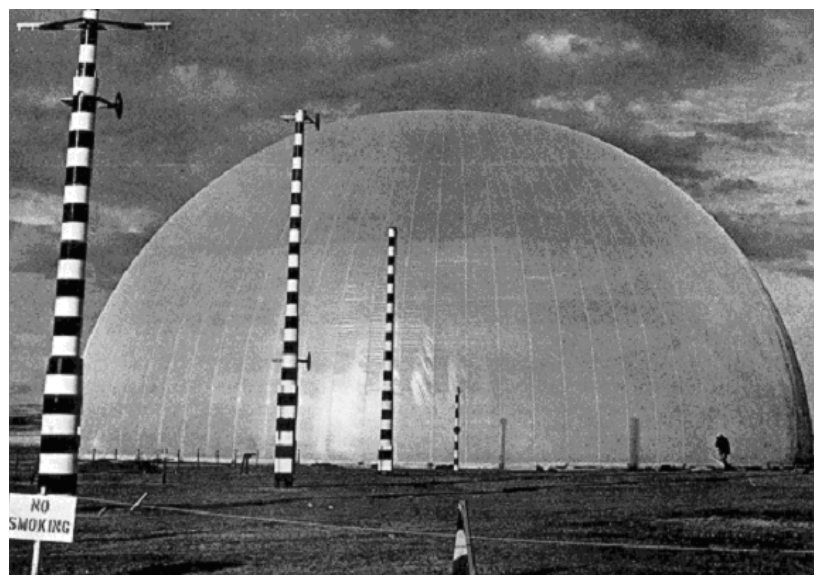

Figure 1. Hemispherical Mylar gas bag filled with a stoichiometric mixture of propane and oxygen. The poles to the left are gun barrels used to carry pressure gauges. They were painted with black and white stripes to provide fiducial markers for the photogrammetric measurements.

\section{Experimental measurements}

Several techniques were applied to measure the blast wave generated by the gaseous explosion, but some were marred by technical failures and all by the displacement, by the wind, of the centre of the gas bag by about $3 \mathrm{~m}$. A number of piezo-electric transducers were deployed, flush mounted with the ground surface, to measure hydrostatic pressuretime histories, and the results were reported by Muirhead and Palmer (1967). Two high-speed photogrammetric methods were used. The refractive image of the primary shock front was photographed against a black and white striped background, and the displacement by the blast of a series of smoke tracers was photographed to provide data for a particle trajectory analysis (Dewey 1971). The analyses of these measurements were reported by Dewey and McMillin (1981). In this case, in addition to the uncertainty about the position of the charge centre, problems were encountered with the accuracy of the timing marks on the films. None of the above measurements, as reported, provides sufficient information to make a reliable calculation of the TNT equivalence over a wide range of distances, and the original data from the photogrammetric measurements are no longer available for re-analysis.

Another measurement technique used on this experiment involved an extensive array of air blast time of arrival detectors (ABTOADs). Each ABTOAD was a simple switch consisting of a brass cylindrical body with a central, insulated and slightly recessed electrode. A silvered Mylar diaphragm was stretched across the end of the cylinder so that its conducting surface was a few millimetres from the end of the central electrode. The device was mounted with the diaphragm pointed towards the centre of the charge. The shock striking the diaphragm caused a contact between the cylinder and the central electrode. A large number of ABTOADs were connected to two wires in a single cable. A voltage difference was maintained between the two wires. As the primary shock of the blast wave reached each ABTOAD, a transitory signal was recorded from the cable and compared with a comb of timing marks. The position of each ABTOAD was surveyed relative to the nominal charge centre and as a result, an accurate record of the times of arrival of the

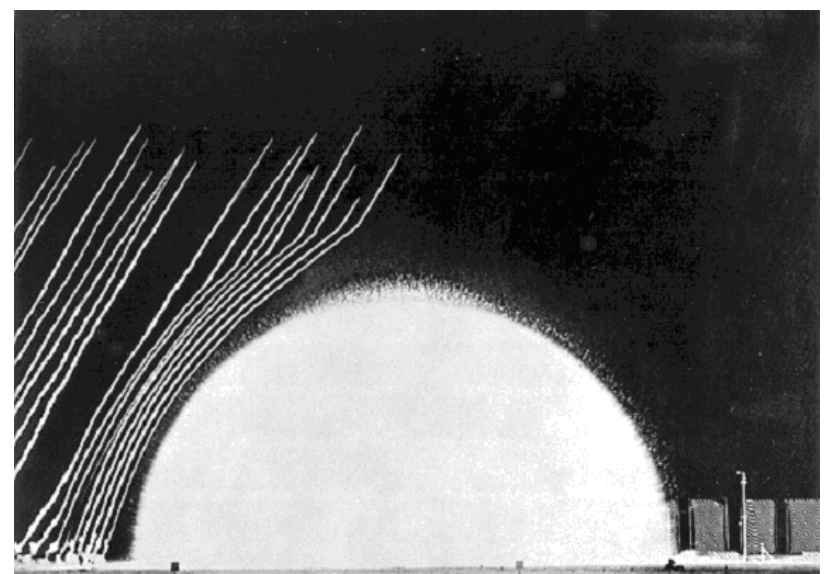

Figure 2. Fireball produced by the propane-oxygen explosion. The white smoke trails were used for the particle trajectory analysis reported by Dewey and McMillin (1981). The discontinuities in the slopes of the trails identify the position of the shock front. The striped canvas screens that provided a background for the refractive image of the shock are in the lower right. The primary shock can be seen passing in front of the second screen from the right.

shock was provided over a wide range of distances. Since only a single cable and two recording channels were required, this constituted a very powerful and cheap measurement technique.

An array of 62 ABTOADs was deployed for the propaneoxygen explosion, at positions ranging from approximately $19 \mathrm{~m}$, i.e. essentially in contact with the gas bag, to $895 \mathrm{~m}$ from the nominal charge centre. The surveyed position of each ABTOAD relative to the displaced charge centre and the recorded times of arrival of the primary shock at each position are reported by Anderson (1970). This appears to be the most reliable set of data from the experiment, covering a range of distances greater than for any other measurement set. The ABTOAD measurements were therefore selected for further analysis.

Figure 2 shows the fireball produced by the explosion, a few milliseconds after detonation. The contact surface between the fireball and the air is uniform, compared with the high degree of turbulent mixing that is caused by detonation of a solid explosive. The initial flash was extremely intense such that the early-time photographs of the event were overexposed. Observers reported that the strong initial thermal radiation could be felt at large distances from the explosion.

\section{Analysis of the ABTOAD data}

The shock radius $(R)$ versus time-of-arrival $(t)$ data from the ABTOAD measurements were scaled, using Sachs scaling, to represent the values that would be expected from the same explosion in an atmosphere at normal temperature and pressure (NTP) (Dewey 2001). The following relationships were used:

$$
\frac{R}{R_{\mathrm{m}}}=\left(\frac{P_{0}}{P_{\mathrm{N}}}\right)^{1 / 3} \quad \text { and } \quad \frac{t}{t_{\mathrm{m}}}=\left(\frac{P_{0}}{P_{\mathrm{N}}}\right)^{1 / 3}\left(\frac{T}{T_{\mathrm{N}}}\right)^{1 / 2}
$$

where $R$ and $R_{\mathrm{m}}$ are the scaled and measured radii, respectively; $t$ and $t_{\mathrm{m}}$ are the scaled and measured times of arrival, respectively; $P_{0}$ is the measured atmospheric pressure 


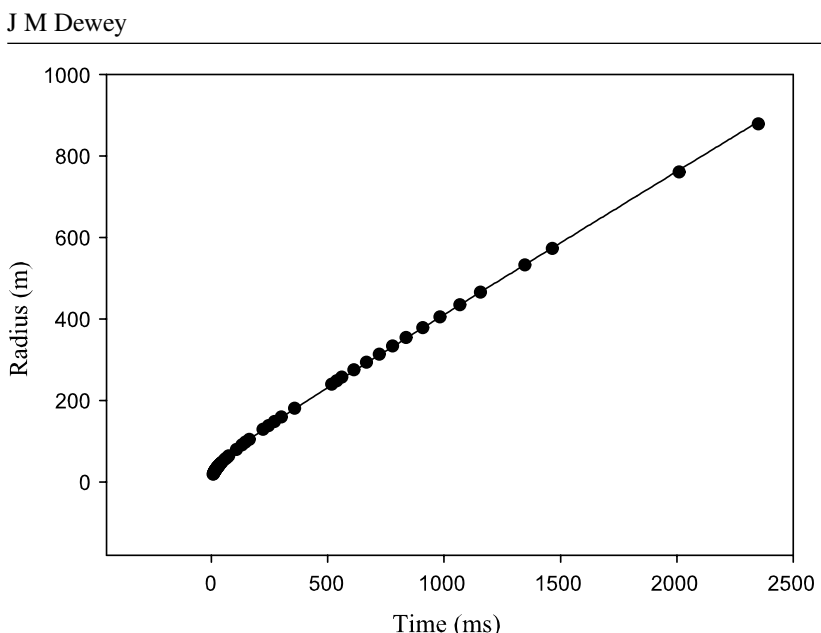

Figure 3. Measured times of arrival of the primary shock (the points), scaled to NTP, and the least squares fit to (2) with no weighting and coefficient $B$ set to one (the curve).

at the time of the explosion $(94.53 \mathrm{kPa}) ; P_{\mathrm{N}}$ is the pressure at NTP $(101.325 \mathrm{kPa}) ; T$ is the atmospheric temperature at a height of $2 \mathrm{~m}$ at the time of the explosion $(296.48 \mathrm{~K})$ and $T_{\mathrm{N}}$ is the temperature at NTP $(288.16 \mathrm{~K})$.

The scaled radius-time data were fitted by least squares to

$$
R=A+B a_{0} t+C \ln \left(1+a_{0} t\right)+D \sqrt{\ln \left(1+a_{0} t\right)}
$$

where $A, B, C$ and $D$ are the fitted coefficients. This equation (Dewey 1971, 2001) has been used extensively to describe the shock radius as a function of time for a large number of centred explosions from different sources and a wide range of charge sizes. Differentiating (2) provides the Mach number of the shock, $M_{\mathrm{S}}$, as a function of time, and therefore of radius, namely

$$
M_{\mathrm{S}}=\frac{1}{a_{0}} \frac{\mathrm{d} R}{\mathrm{~d} t}=B+\frac{C}{1+a_{0} t}+\frac{D}{2\left(1+a_{0} t\right) \sqrt{\ln \left(1+a_{0} t\right)}} .
$$

The values of $M_{\mathrm{S}}$ obtained from (3) were used in the RankineHugoniot relationship,

$$
\frac{\mathrm{OP}_{\mathrm{S}}}{P_{\mathrm{N}}}=\frac{7}{6}\left(M_{\mathrm{S}}^{2}-1\right)
$$

to obtain the hydrostatic overpressure, $\mathrm{OP}_{\mathrm{S}}$, behind the primary shock in terms of the atmospheric pressure at NTP, $P_{\mathrm{N}}$, assuming a ratio of specific heats for air of $\gamma=1.4$.

In fitting the measured times-of-arrival data to (2), the coefficient $B$ was held constant at one. This ensured that, as the primary shock, and the corresponding time $t$ in (3), increased to infinity, the shock speed asymptotically approached the ambient sound speed. The measured data and the fitted curve are shown in figure 3.

The shock-radii, from the closest to the furthest, varied by a factor of approximately 50 . As a result, the least-squares fit to (2) proportionally favours the data at large radii. To compensate for this, fits were also made using weighting factors of $1 / R$ and $1 / R^{2}$. A review of the residuals of these fits shows, as is to be expected, that the fit with no weighting has the smallest residuals at large radii and the fit with $1 / R^{2}$ weighting has the smallest residuals at the shortest shock radii. The coefficients from these two fits were used in (3) to provide

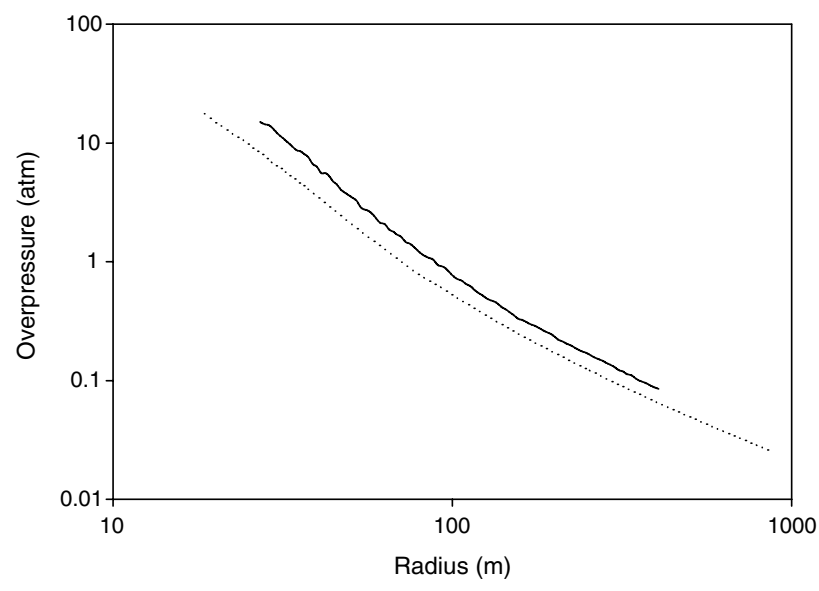

Figure 4. Overpressure versus radius at NTP for the propane-oxygen mixture $(\cdots \cdots)$ and for 20 short tons of TNT, surface burst, obtained from AirBlast (- - ).

the shock Mach number as a function of distance. The resulting Mach numbers were used in (4) to obtain the variation of overpressure with distance, in each case.

To obtain a weighted mean between these two data sets, the following averaging formula was used:

$$
P_{n}=\frac{P_{1 n} \cdot R_{N-n}-P_{2 n} \cdot R_{n}}{R_{N-n}+R_{n}},
$$

where $P_{n}$ is the weighted mean of the $n$th overpressure, $P_{1 n}$ and $P_{2 n}$ are the $n$th overpressures of the two data sets, respectively, $N$ is the total number of values in each of the data sets and $R_{n}$ and $R_{N-n}$ are the radii of the $n$th and $(N-n)$ th data values, respectively. This has the effect of favouring the $P_{1}$ data set at small radii and the $P_{2}$ data set at large radii. The resulting weighted mean overpressure as a function of radius is plotted in figure 4, and these data were used in the subsequent analysis.

The overpressure versus radius data, which had already been scaled to an ambient atmosphere at NTP, were then scaled to a unit charge mass using

$$
\frac{R_{\mathrm{S}}}{R}=\left(\frac{1}{W}\right)^{1 / 3},
$$

where $R_{\mathrm{S}}$ and $R$ are the scaled and observed radii, respectively, and $W$ is the mass of the explosive charge (Dewey 2001).

In the case of the propane-oxygen explosion, there were two possible charge masses: the total mass of the propane and oxygen and the mass of the propane alone. The latter might better represent a mass of propane that had been dispersed to form an optimum vapour-air mixture before detonation. The data derived from (5) were scaled to represent the results from a charge of unit mass, using (6) with $W$ equal to $19281 \mathrm{~kg}$, the mass of the propane and oxygen, and $5447 \mathrm{~kg}$, the mass of the propane alone. The resulting overpressures, as functions of scaled radius, are plotted in figure 5 and compared with the values for a $1 \mathrm{~kg}$ hemispherical, surface-burst charge of TNT obtained from AirBlast ${ }^{1}$.

1 AirBlast ${ }^{\circledR}$ is an expert system that provides the physical properties of blast waves from TNT, based on a large number of experimental measurements. It is a proprietary software of Dewey McMillin \& Associates Ltd (www.blastanalysis.com) and JASCO Research Ltd (www.jasco.com). 


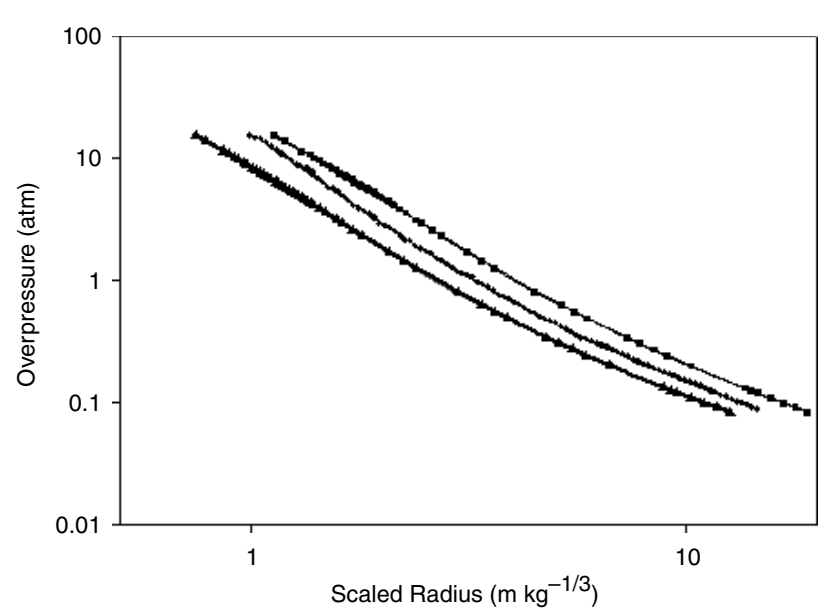

Figure 5. Overpressure versus scaled radius for the propane-oxygen mixture $(\boldsymbol{\Delta})$, propane alone $(\boldsymbol{\square})$ and TNT $(\bullet)$. The curves are the least squares fits to (7) with the coefficients listed in table 1 .

Table 1. Fitted coefficients to equation (7)

\begin{tabular}{lllll}
\hline & $E$ & $F$ & $G$ & $H$ \\
\hline Propane/oxygen & 0.9835 & -0.4907 & 0.0315 & $-8.6210 \mathrm{e}-3$ \\
Propane & 1.4048 & -0.4907 & 0.0318 & $-8.6211 \mathrm{e}-3$ \\
TNT & 1.1778 & -0.4887 & 0.0400 & $-5.9501 \mathrm{e}-3$ \\
\hline
\end{tabular}

The three sets of overpressure and scaled radius data were fitted by least squares to the equation

$$
\ln R_{\mathrm{S}}=E+F \ln P+G(\ln P)^{2}+H(\ln P)^{3},
$$

where $E, F, G$ and $H$ are the fitted coefficients. A function of the form $R_{\mathrm{S}}=f(P)$ was used, rather than $P=f\left(R_{\mathrm{S}}\right)$, to facilitate the subsequent analysis. There is no physical significance to the form of (7), but experience has shown that it is a useful relationship to describe the radius of a blast wave in terms of the peak overpressure. The curves through the data points in figure 5 are the least squares fits to (7). The fitted coefficients for the propane plus oxygen, the propane alone and TNT are given in table 1.

The TNT equivalence of an explosive is defined as the mass of the explosive that will produce a blast wave with the same strength, peak hydrostatic overpressure in this case, as a unit charge of TNT at the same radial distance. Therefore, using Hopkinson scaling (Dewey 2001), the TNT equivalence, $\eta$, is given by

$$
\eta=\frac{W_{\mathrm{E}}}{1}=\left(\frac{R_{\mathrm{E}}}{R_{\mathrm{T}}}\right)^{3},
$$

where $W_{\mathrm{E}}$ is the equivalent charge mass, and $R_{\mathrm{E}}$ and $R_{\mathrm{T}}$ are the distances from the studied explosive and TNT, respectively, at which the same peak overpressure was observed. Normally, $\eta$ is not a constant value but varies with the overpressure. Using (8) $\eta$ can be written as a function of overpressure, namely

$$
\eta(P)=\left[\frac{R_{\mathrm{E}}(P)}{R_{\mathrm{T}}(P)}\right]^{3},
$$

where $R(P)$ is given by (7) as

$$
R(P)=\exp \left[E+F \ln P+G(\ln P)^{2}+H(\ln P)^{3}\right],
$$

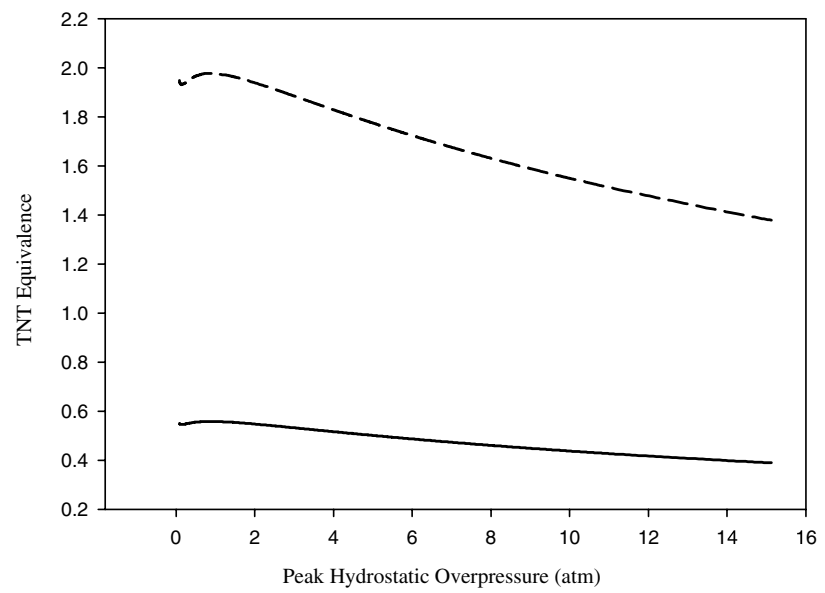

Figure 6. TNT equivalences as a function of overpressure for propane-oxygen (-) and propane alone (- - -).

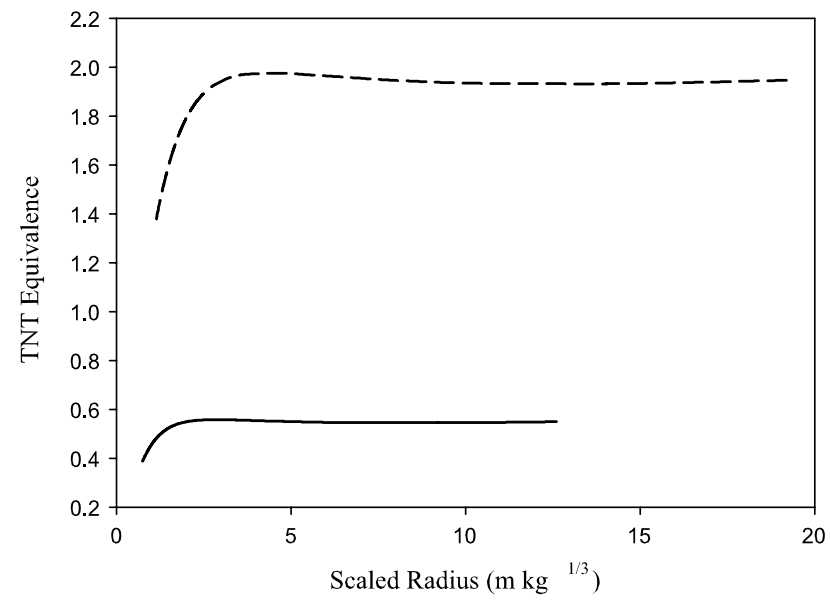

Figure 7. TNT equivalences as a function of scaled radius for propane-oxygen ( - ) and propane alone (- - -).

Table 2. Fitted coefficients of equation (11).

\begin{tabular}{lllll}
\hline & $J$ & $K$ & $L$ & $M$ \\
\hline Propane/oxygen & 0.4580 & 0.2150 & -0.1442 & 0.0292 \\
Propane & 1.2020 & 1.2462 & -0.6410 & 0.1034 \\
\hline
\end{tabular}

and the coefficients for the explosives, propane/oxygen, propane alone and TNT are given in table 1.

The values of the TNT equivalences as functions of overpressure for propane/oxygen and propane, derived using (9) and (10), are plotted in figure 6. Alternatively, the equivalences can be plotted as functions of distance from a unit charge, as shown in figure 7. To simplify the use of these relationships, it was found that they could be described by least squares fits to log-cubic functions of the form

$$
\eta=J+K \ln R_{\mathrm{S}}+L\left(\ln R_{\mathrm{S}}\right)^{2}+M\left(\ln R_{\mathrm{S}}\right)^{3},
$$

where $J, K, L$ and $M$ are the fitted coefficients and $R_{\mathrm{S}}$ is the scaled radius, i.e. the distance from a $1 \mathrm{~kg}$ charge. The values of the fitted coefficients are given in table 2 . 


\section{Discussion and conclusions}

The ABTOAD measurements analysed here were chosen because they provide a unique data set describing a physical property of a blast wave, produced by a well-defined hemispherical gaseous explosive source, over a wide range of distances from immediately adjacent to the source to the region in which the peak overpressure had decayed to about $0.05 \mathrm{~atm}$. Measurements of other gaseous explosions did not appear to be so unambiguous, usually did not cover such a wide range of distances and were not produced by such a well-defined hemispherical or spherical explosive source. The principal source of error in the measurements that were used arose from the uncertainty of the position of the centre of the explosion, which may have been displaced by up to $3 \mathrm{~m}$.

The measured shock-radius versus time-of-arrival data were fitted by least squares to equation (2). This equation has been used successfully for many years to describe trajectories of the primary shock fronts from hemispherical and spherical explosive sources. It is monotonic and its derivative asymptotically approaches a constant value, the ambient sound speed, as time approaches infinity. Polynomial forms of equations cannot be used for this purpose because their derivatives, i.e. the shock speed, are oscillatory.

The shock radii fitted to (2) cover a range that varies by a factor of 50. As a result, the unweighted fit is proportionally better at large values of the shock radius. To compensate for this, fits were also made using weighting factors of $1 / R$ and $1 / R^{2}$. The fitted equations were differentiated to provide the shock speed and thus the shock Mach number and peak overpressure as functions of radius and time. A weighted average of the overpressures obtained from the unweighted and the $1 / R^{2}$ weighted fits were calculated. It is believed that this provides the best possible description of the variation of peak overpressure as a function of distance for this explosion.

A precise measure of the amounts of propane and oxygen used in the source was not reported, other than that it was a nominal 20 ton charge. The amounts of propane and oxygen were therefore calculated from the available information and indicate a total charge mass of $19281 \mathrm{~kg}(21.25 \mathrm{tn})$ comprised of $5447 \mathrm{~kg}$ of propane and $13834 \mathrm{~kg}$ of oxygen. The overpressure versus distance data were scaled to unit charges using both these charge masses. The scaled data were compared with the overpressure versus distance relationship for a unit, hemispherical, surface-burst TNT charge to obtain the TNT equivalence as a function of overpressure.

The equivalence factors versus overpressure are plotted in figure 6. The form of these relationships, each with a maximum, was to have been expected based on the work of Strehlow et al (1979). These authors use a numerical calculation to determine the variation of overpressure with scaled distance for gaseous mixtures with different deflagration velocities. These variations were compared with that for Pentolite. If their results for high deflagration speeds, say, Mach 2, and Pentolite were to be analysed in the same way as those for propane/oxygen and TNT above, relationships similar to those shown in figures 6 and 7 would be obtained.

In figure 7 the equivalence factors are plotted as functions of radius from a charge of unit mass. At small radii, immediately adjacent to the surface of the gaseous charge, the overpressure is much less than that which would be produced by an explosion of the same mass of TNT. As the shock front expands, its strength increases relative to that from TNT. Eventually a distance is reached, about $2 \mathrm{~m} \mathrm{~kg}^{-1 / 3}$ for propaneoxygen and $4 \mathrm{~m} \mathrm{~kg}^{-1 / 3}$ for propane, beyond which the blast wave from the gaseous explosion decays in a manner very similar to that from TNT. If the curves in figure 6 are plotted on a larger scale, it can be seen that there is a minimum and a maximum between zero and 2 atm overpressure. It is not known whether this slightly oscillatory form of the relationship at larger radii is real, but such a form is not improbable. It suggests that the shock wave from the gaseous explosion, compared with that from a fixed mass of TNT, at first overtakes the shock wave from TNT, moves ahead, slightly recedes and then overtakes again.

The TNT equivalence versus scaled distance relationships, shown in figure 7 , show that it is invalid to use a single TNT equivalence factor for a gaseous explosion in the region of overpressures greater than about $1 \mathrm{~atm}(100 \mathrm{kPa}, 15 \mathrm{psi})$. At lower overpressures a single equivalence factor is valid: 0.55 for a total charge mass including the propane and oxygen and 1.95 for a charge mass including the propane only and assuming an optimum mixture of the propane with atmospheric oxygen. This confirms the conclusions of Dewey and McMillin (1981), who found that for peak overpressures less than about $1.5 \mathrm{~atm}$ the wave profiles of the blast wave from the propane-oxygen explosion were similar to those produced by a hemispherical $20 \mathrm{tn}$ TNT charge.

The TNT equivalence of VCE is well described by Lee et al (1977), who define the explosive yield of TNT in terms of its nominal explosive energy of $4.198 \times 10^{6} \mathrm{~J} \mathrm{~kg}^{-1}$. In this paper, no assumption is made about the energy yield of TNT, but a direct comparison is made with the blast wave from a hemispherical charge. In the above reference, in response to a question, Lee points out the difficulty of assigning a single number to the TNT equivalence of a VCE, particularly at short distances from the source. The present work suggests that a single equivalence factor may be valid at distances corresponding to overpressures less than about $1 \mathrm{~atm}$. The value of that factor will depend on other circumstances such as the degree of mixing of the fuel and oxygen and the shape of the vapour cloud. However, the present results would appear to provide an upper limit for an optimum propane-oxygen mixture.

In the case of a BLEVE, optimum mixing of the propane vapour and droplets with atmospheric oxygen cannot be assumed. In that case, it would be appropriate to reduce the amount of propane by a suitable factor before calculating the physical properties of the blast wave produced by the gaseous detonation. Baker et al (1983) and Birk (1996) both suggest using a factor of $10 \%$. If this factor were applied to the equivalence factor of 1.95 , based on the mass of propane alone in the propane-oxygen explosion, this would suggest a TNT equivalence of $20 \%$ for the mass of propane involved in a BLEVE. The unconsumed propane may continue to burn and produce an extended outward flow, but this is unlikely to have any significant damaging effect compared with that produced by the initial pressure explosion or the subsequent fuel-air detonation/deflagration. It is hoped that reliable measurements of blast properties at known distances from VCE produced by BLEVEs will lead to a better estimate of the reduction factor. 
The difference between the TNT equivalence factors of 1.95 , using the mass of propane alone, and of 0.55 , using the total mass of the propane and oxygen, illustrates the principle of so-called thermo-baric explosives that are used in some modern weapon systems. An explosive such as TNT relies on the oxygen within its chemical composition to release its energy during the detonation process. Using a non-oxygenated fuel, such as propane, which can be dispersed to mix with atmospheric oxygen for its energy release, means that a weapon of approximately half the size can be used to produce a similar blast wave. Less damage will be caused close to the dispersed explosion, but similar, or even more, damage to that caused by the equivalent mass of TNT will occur at peak overpressure levels less than about $1 \mathrm{~atm}$.

\section{Acknowledgments}

The assistance of C Needham, D Harrington and K Dixon in determining the masses of propane and oxygen in the charge is gratefully acknowledged.

\section{References}

Anderson J H B 1970 Canadian air blast measurements from Operation 'DISTANT PLAIN' Event 2A Suffield Technical Note No 270 Defence Research Establishment Suffield, Ralston, AB, Canada

Martinsen W E and Melton T A 1999a A comparison of vapor cloud explosion models Quest Q. 4 1-4 http://www.questconsult.com

Martinsen W E and Melton T A 1999b A comparison of vapor cloud explosion models-Part II Quest Q. 4 1-4 http://www.questconsult.com
Baker W E, Cox P A, Westine P S, Kulesz J J and Strehlow R A 1983 Explosion Hazards and Evaluation (Amsterdam: Elsevier)

Birk A M 1996 Hazards from propane BLEVEs: an update and proposal for emergency responders J. Loss Prevent. Process Indust. 9 173-81

Birk A M, Ostic P, Cunningham M, Kielec D and Miller T 1995 Fire test of propane tanks to study BLEVEs and other thermal ruptures: detailed analysis of medium scale results Transport Canada Report TP12498E (Ottawa: Canadian Institute for Science and Technical Information)

Cooper P W 1996 Explosives Engineering (New York: Wiley)

Dewey J M 1971 The properties of a blast wave obtained from an analysis of the particle trajectories Proc. R. Soc. Lond. A 324 275-99

Dewey J M 2001 Expanding spherical shocks (blast waves) Handbook of Shock Waves vol 2, ed G Ben-Dor et al (San Diego: Academic) pp 441-81, chapter 13.1

Dewey J M and McMillin D J 1981 The properties of a blast wave produced by a large-scale detonable gas explosion Proc. 7th Int. Symp. on Military Applications of Blast Simulation (Defence Research Establishment Suffield, Canada) vol II, paper 6.6, pp 1-18

Kleine H, Dewey J M, Ohashi K, Mizukaki T and Takayama K 2003 Studies of the TNT equivalence of silver azide charges Shock Waves 13 123-38

Lee J H, Guirao C M, Chiu K W and Bach G G 1977 Blast effects from vapor cloud explosions Loss Prevent. 2 59-70

Muirhead J C and Palmer W O 1967 Canadian participation in distant plain (Shots 1, 2, 2a and 3): air blast pressure gauge measurements Suffield Technical Note 177, Defence Research Establishment Suffield, Ralston, AB, Canada

Strehlow R A, Luckritz R T, Adamczyk A A and Shimpi S A 1979 The blast wave generated by spherical flames Combust. Flame 35 297-310

Van den Berg A C, Van Wingerden C J M and The H G 1991 Vapour cloud explosions: experimental investigation of key parameters and blast modelling Trans. IchemE B 69 139-48 